OPEN ACCESS

Edited by:

Jun Zhang,

University of Kansas Medical Center,

United States

Reviewed by:

Chao H Huang,

University of Kansas Medical Center

Research Institute, United States

Haitang Yang,

Shanghai Jiaotong University, China

*Correspondence:

Seung Hyeun Lee humanmd04@hanmail.net

Specialty section: This article was submitted to Thoracic Oncology,

a section of the journal

Frontiers in Oncology

Received: 03 September 2021 Accepted: 22 November 2021 Published: 09 December 2021

Citation:

Kwack WG, Sung J-Y and Lee SH (2021) Overexpression of Reactive Oxygen Species Modulator 1 Predicts

Unfavorable Clinical Outcome in EGFR-Mutant Lung Adenocarcinomas Treated With Targeted Therapy.

Front. Oncol. 11:770230. doi: 10.3389/fonc. 2021.770230

\section{Overexpression of Reactive Oxygen Species Modulator 1 Predicts Unfavorable Clinical Outcome in EGFR-Mutant Lung Adenocarcinomas Treated With Targeted Therapy}

\author{
Won Gun Kwack ${ }^{1}$, Ji-Youn Sung ${ }^{2}$ and Seung Hyeun Lee ${ }^{1 *}$ \\ ${ }^{1}$ Division of Pulmonary, Allergy, and Critical Care Medicine, Department of Internal Medicine, College of Medicine, Kyung \\ Hee University, Seoul, South Korea, ${ }^{2}$ Department of Pathology, College of Medicine, Kyung Hee University, \\ Seoul, South Korea
}

Purpose: Reactive oxygen species modulator 1 (Romo1) is a novel protein that regulates the production of intracellular reactive oxygen species. Romo1 has been shown to be associated with poor survival in various clinical settings for the treatment of lung cancer. In this study, we evaluated whether tissue Romo1 expression was associated with clinical outcomes in epidermal growth factor receptor (EGFR)-mutated lung adenocarcinoma treated with tyrosine kinase inhibitors (TKIs).

Method: Romo1 expression in tumor tissues was examined by immunohistochemistry and evaluated by histologic score. Univariate and multivariate analyses were performed to identify the clinicopathologic parameters, including Romo1 expression, which may be associated with progression-free survival (PFS), overall survival (OS), and incidence of secondary T790M mutation.

Results: A total of 96 tumor specimens were analyzed. With the cut-off value of 200, 71 (74.0\%) and 25 (26.0\%) patients were classified into low and high Romo1 groups, respectively. The median PFS of the high Romo1 group was significantly shorter than that of the low Romo1 group (13.1 vs 19.9 months, $p=0.0165$ ). The median OS of the high Romo1 group was also significantly shorter than that of the low Romo1 group (19.8 vs 37.0 months, $p=0.0006$ ). Multivariate analyses showed that high Romo1 expression was independently associated with both poor PFS (hazard ratio $[\mathrm{HR}]=2.48,95 \%$ confidence interval $[\mathrm{Cl}]$ : $1.35-4.56, p=0.0034)$ and poor OS (HR $=3.17,95 \% \mathrm{Cl}: 1.57-6.41, p=$ 0.0013). In addition, the rate of secondary T790M mutation after TKI failure was significantly lower in the high Romo1 group than the low Romo1 group (16.7\% vs. $38.3 \%, p=0.0369$ ).

Conclusions: Romo1 overexpression was associated with poor response to treatment and short survival in patients treated with EGFR-TKIs, suggesting a distinct subgroup 
warranting active surveillance and tailored therapeutic approach. In addition, our data highlight that Romo1 could be a potential predictive and prognostic biomarker for this patient population.

Keywords: reactive oxygen species modulator 1, biomarker, survival, epidermal growth factor receptor, targeted therapy

\section{INTRODUCTION}

Lung cancer has been the leading cause of cancer-related deaths worldwide. Globally, it accounted for 2.2 million new cases and 1.8 million deaths in 2020 (1). Adenocarcinoma is the dominant histologic subtype, accounting for $60 \%$ of non-small cell lung cancers (NSCLCs) (2). Although recent advances in treatment modalities, including targeted therapy and immunotherapy, have demonstrated better clinical outcomes than conventional chemotherapy in patients with advanced lung adenocarcinoma $(2,3)$, the mortality of this lethal disease is still high, with a 5-year survival rate of only $24 \%$ (4), suggesting the unmet clinical need for personalized therapeutic approaches to improve prognosis.

Reactive oxygen species modulator 1 (Romo1) was first identified in 2006 in a patient with head and neck cancer who was resistant to chemotherapy after recurrence (5). Located in the mitochondrial membrane, this novel protein is a major regulator of the production of intracellular reactive oxygen species (ROS), and Romo1-induced ROS play a significant role in cell proliferation in both cancer and normal cells $(6,7)$. In addition, Romo1-induced ROS are related to the drug resistance of 5-fluorouracil (5-FU), and Romol overexpression induces invasive activity in various cancer cells $(8-10)$. These findings suggest that Romol may not only be involved in carcinogenesis but may also influence the response to anticancer treatment. This concept has been proven using recent clinical data $(9,11-13)$. Chung et al. demonstrated that tumor Romol overexpression was significantly associated with poor survival and vascular invasion in patients with hepatocellular carcinoma who underwent curative resection (9). Lee et al. demonstrated that high Romol expression was significantly associated with early recurrence and poor survival after surgical resection in patients with NSCLC (11). Romol overexpression in tumor tissue was significantly associated with poor response and clinical outcomes in patients with advanced NSCLC treated with platinum doublets (12). In addition, Kim et al. reported that elevated Romo1 expression in curatively resected colorectal cancer was significantly associated with poor survival outcomes and frequent lymph node metastasis (13). These data suggest that Romol is a promising biomarker for malignancies. However, the clinical usefulness of this protein has never been explored in patients with cancer harboring driver genetic alterations.

In this study, we aimed to determine the clinical implications of Romo1 expression in patients with epidermal growth factor receptor (EGFR)-mutant lung adenocarcinoma treated with first-line tyrosine kinase inhibitors (TKIs). We investigated whether Romol expression is related with the response to treatment, survival rate, and the development of secondary T790M mutations after TKI failure.

\section{MATERIALS AND METHODS}

\section{Study Subjects and Data Collection}

We retrospectively enrolled EGFR-mutant patients treated with first-line EGFR-TKIs for locally advanced or metastatic lung adenocarcinoma at Kyung Hee University Hospital, a referral hospital in South Korea, from April 2016 to March 2020. Patients without insufficient survival data, history of other malignancies, or other driving genetic alterations, including ROS protooncogene 1 (ROS1) and anaplastic lymphoma kinase (ALK) fusions, and those with a history of previous chemo- or radiotherapy were excluded from the study.

Staging workup was performed using chest computed tomographic (CT) scan, brain magnetic resonance imaging, and ${ }^{18} \mathrm{~F}$-fluorodeoxyglucose positron emission tomographycomputed tomography. Tumor-node-metastasis (TNM) staging was evaluated based on the eighth edition of the American Joint Commission on Cancer TNM staging system of NSCLC (14). After every two cycles of systemic treatment, tumor response was assessed with chest CT scan according to the Response Evaluation Criteria in Solid Tumors (RECIST) 1.1 (15). Electronic medical records were reviewed to collect patient demographics, medical or social history, and survival data. The study protocol was reviewed and approved by the Clinical Research Ethics Committee of Kyung Hee University Hospital (KHUH 2020-03-026). We obtained written informed consent from all participants who were alive. All studies were conducted in compliance with the Declaration of Helsinki.

\section{Romo1 Immunohistochemical Staining and Scoring}

Romol protein expression was evaluated by immunohistochemical (IHC) staining. We used a BOND-MAX Immunoautostainer (Leica Biosystems, Newcastle, UK) of the staining after preparation of 4micron-thick sections of paraffin-embedded tumor tissues. Antigen retrieval was performed by heating the slides at $98^{\circ} \mathrm{C}$ for $20 \mathrm{~min}$ and cooling them for $10 \mathrm{~min}$ in Epitope Retrieval Solution 1 and $0.01 \mathrm{M}$ citrate buffer ( $\mathrm{pH} 6.0$ ), respectively. Slides were then washed in distilled water and endogenous peroxidase activity was blocked using a Bond Polymer Refine Detection Kit (Leica Biosystems, Newcastle, UK) for $5 \mathrm{~min}$. After washing, the slides were placed in Tris-buffered saline and incubated for $30 \mathrm{~min}$ with Romo1 monoclonal antibody (OriGene Technologies, Rockville, USA) at 1:200 dilution. Subsequently, sections were developed with 3,3'diaminobenzidine chromogen solution for $7 \mathrm{~min}$, counterstained with hematoxylin, and dehydrated. Positive and negative controls were defined using human colon adenocarcinoma tissues and exclusion of the primary antibody, respectively. 
Blinded to the clinical information, a pathologist (J-Y Sung) accessed the Romol staining intensities under a light microscope at $200 \mathrm{x}$. Stained cells were considered as positive when the cytoplasmic staining was identified. Staining intensities of individual cells were graded as 0 (no staining), 1 (weak), 2 (distinct), or 3 (strong), and the percentages of cells with these staining intensities were calculated. Finally, histological scores $(\mathrm{H}$ scores) were calculated by multiplying the staining intensities by percentages of cells with each staining intensity (possible range, 0-300).

\section{EGFR Mutation Testing}

EGFR mutation tests were performed using tumor tissues. Genomic DNA was extracted from formalin-fixed, paraffinembedded, 5- $\mu$ m-thick tissue sections using the High Pure Template Preparation Kit (Roche Applied Science, Mannheim, Germany). The extracted DNA was stored at $-20{ }^{\circ} \mathrm{C}$ until analysis. PANAMutyper $^{\mathrm{TM}}$ (PANAGENE Inc., Daejeon, South Korea), a PNA-Clamping-based EGFR mutation detection kit, was used for the detection of EGFR mutations. The primer sets covered mutations or deletions spanning exons 18-21 of the genes encoding the tyrosine kinase domain of EGFR. Results were interpreted according to the manufacturer's instructions.

\section{Statistical Analyses}

The cut-off $\mathrm{H}$ score for discriminating between low and high Romol expression was defined as the point with the lowest $p$ value by the log-rank test for all possible $\mathrm{H}$ scores. Baseline characteristics of different groups were compared using the Chisquare test or Fisher's exact test, as appropriate. Clinical outcomes including the response rate (RR), progression-free survival (PFS), and overall survival (OS) were assessed. RR was defined as the percentage of patients who achieved complete or partial response. PFS was defined as the period from the first day of treatment to disease progression or death. OS was defined as the interval from the first day of treatment to death from any cause. Data of patients without tumor recurrence or death were censored at the last follow-up. Correlations between survival outcomes and clinicopathological parameters were estimated by univariate analysis using the log-rank test, followed by Cox proportional hazard regression analysis. Parameters with $p$ values $<0.1$ in the univariate analysis were included for the multivariate analysis. Kaplan-Meier method was used to estimate survival rates. $p<0.05$ was considered as statistically significant. All analyses were performed using SPSS v.20.0 (IBM Corporation, Armonk, NY, USA).

\section{RESULTS}

\section{Patient Characteristics}

During the study period, 718 patients were newly diagnosed with NSCLC, and 259 patients were diagnosed with advanced lung adenocarcinoma. Of these patients, 121 received EGFR-TKIs as a frontline treatment for EGFR-positive diseases. Thirteen patients with insufficient survival data, eight with concomitant cancers, and four who received other cancer treatments before targeted therapy were excluded. Finally, 96 patients were included in the analysis.

The clinical characteristics of the study population are summarized in Table 1. All subjects were Korean, and their median age was 69 years (range, 41-88 years). Fifty-three (55.2\%) patients were aged $\geq 70$ years, 51 (53.1\%) patients were women, and $32(33.3 \%)$ patients were current or former smokers. Seventy-five $(78.1 \%)$ patients had an Eastern Cooperative Oncology Group performance status (ECOG PS) of 0 or 1. Sixteen (16.7\%) patients had stage III disease, and $80(83.3 \%)$ patients had stage IV disease. Twenty-three (24.0\%) had metastases involving three or more organs, while $22(34.4 \%)$ and $11(11.5 \%)$ patients had brain or liver metastases, respectively. Fifty-two (54.1\%) patients had exon 19 deletion (19del), 39 (40.6\%) had L858R point mutations, and 5 (5.2\%) had uncommon or compound mutations. Seventy- two $(74.8 \%)$ patients received afatinib, while $24(25.2 \%)$ treated with gefitinib or erlotinib as a frontline therapy.

\section{Romo1 Protein Expression}

Representative sections with different $\mathrm{H}$ scores are shown in Figure 1. As Romol is located in the mitochondrial membrane, it was primarily localized in the cytoplasm of cancer cells, as expected. The median $\mathrm{H}$ score of the study population was 160.0 (range, 65-290).

\section{Association Between Clinicopathological Parameters and Romo1 Expression}

To investigate which clinicopathological parameters were associated with the level of Romol expression, we compared the median Romol H score between groups within each parameter. The median Romol $\mathrm{H}$ score was significantly higher in patients with a more advanced stage (160 vs. 135, $p=0.0443$, Supplementary Table 1). We then compared the distribution of patients according to the Romol group. The optimal cut-off value for low and high Romol expression was determined to be $200 \mathrm{H}$ score by the log rank test. Using this cutoff, $56(72.7 \%)$ patients and $21(27.3 \%)$ patients were classified to the low and high Romol groups, respectively. As shown in Table 1, Romol expression was not related to any parameters, including age, sex, smoking history, ECOG PS, and EGFR subtype. However, high Romol expression tended to be associated with a more advanced stage $(p=0.0747)$.

\section{Response Rate and PFS According to Romo1 Expression}

The median follow-up period for our study subjects was 28.9 months (range, 3.1-56.0 months). The treatment response according to Romol expression is summarized in Table 2. In the low Romol group, one (1.4\%) and 59 (83.1\%) patients showed complete response (CR) and partial response (PR), respectively, while 17 (68\%) patients showed PR in the high Romol group. The RR was significantly lower in the high Romol group than in the low Romo1 group ( $68.0 \%$ vs. $84.5 \%, p=0.0447)$.

Table 3 shows the PFS analysis results according to clinicopathological parameters. Seventy-seven patients $(80.2 \%)$ 
TABLE 1 | Characteristics of 96 study patients stratified by Romo1 expression levels.

\begin{tabular}{|c|c|c|c|c|}
\hline & \multirow[t]{2}{*}{ No. of patients (\%) } & \multicolumn{2}{|c|}{ Romo1 H score } & \multirow[t]{2}{*}{$p$-value } \\
\hline & & Low $(<200, n=71)$ & High $(\geq 200, n=25)$ & \\
\hline \multicolumn{5}{|l|}{ Age } \\
\hline$<70$ & $43(44.8)$ & $34(47.9)$ & $9(36.0)$ & 0.3040 \\
\hline$\geq 70$ & $53(55.2)$ & $37(52.1)$ & $16(64.0)$ & \\
\hline \multicolumn{5}{|l|}{ Sex } \\
\hline Male & $45(46.9)$ & $32(45.1)$ & $13(52.0)$ & 0.5504 \\
\hline Female & $51(53.1)$ & $39(54.9)$ & $12(48.0)$ & \\
\hline \multicolumn{5}{|l|}{ Smoking } \\
\hline Never & $64(66.7)$ & $47(66.2)$ & $17(68.0)$ & 0.8694 \\
\hline Ever & 32 (33.3) & $24(33.8)$ & 8 (32.0) & \\
\hline \multicolumn{5}{|l|}{ Smoking intensity } \\
\hline <30 pack-years & 77 (80.2) & $56(78.9)$ & $21(84.0)$ & 0.7722 \\
\hline$\geq 30$ pack-years & 19 (19.8) & $15(21.1)$ & $4(16.0)$ & \\
\hline \multicolumn{5}{|l|}{ ECOG PS } \\
\hline 0,1 & $75(78.1)$ & $55(77.5)$ & $20(80.0)$ & 0.7920 \\
\hline$\geq 2$ & $21(21.9)$ & $16(22.5)$ & $5(20.0)$ & \\
\hline \multicolumn{5}{|l|}{ Stage } \\
\hline III & $16(16.7)$ & $14(19.7)$ & $2(8.0)$ & 0.0747 \\
\hline IV & 80 (83.3) & 57 (80.3) & $23(92.0)$ & \\
\hline \multicolumn{5}{|l|}{ Involved organ } \\
\hline$<3$ & $73(76.0)$ & $53(74.6)$ & $20(80.0)$ & 0.5898 \\
\hline$\geq 3$ & $23(24.0)$ & $18(25.4)$ & $5(20.0)$ & \\
\hline \multicolumn{5}{|l|}{ Brain metastasis } \\
\hline No & $63(65.6)$ & 47 (66.2) & $16(64.0)$ & 0.8423 \\
\hline Yes & $33(34.4)$ & $24(33.8)$ & $9(36.0)$ & \\
\hline \multicolumn{5}{|l|}{ Liver metastasis } \\
\hline No & 85 (88.5) & 62 (87.3) & $23(92.0)$ & 0.7225 \\
\hline Yes & $11(11.5)$ & $9(12.7)$ & $2(8.0)$ & \\
\hline \multicolumn{5}{|l|}{ EGFR subtypes } \\
\hline 19del & $52(54.1)$ & $38(53.5)$ & $14(56.0)$ & 0.1921 \\
\hline L858R & $39(40.6)$ & $28(39.4)$ & $11(44.0)$ & \\
\hline Others & $5(5.2)$ & $5(2.8)$ & $0(0.0)$ & \\
\hline First-line TKI & & & & 0.3475 \\
\hline Gefitinib & $16(16.8)$ & $10(14.1)$ & $6(24.0)$ & \\
\hline Erlotinib & $8(8.4)$ & $4(5.6)$ & $4(16.0)$ & \\
\hline Afatinib & $72(74.8)$ & 57 (80.3) & $15(60.0)$ & \\
\hline
\end{tabular}

ECOG PS, Eastern Cooperative Oncology Group Performance Status; EGFR, epidermal growth factor receptor; 19del, deletion mutation at exon 19; TKI, tyrosine kinase inhibitor.

progressed during the follow-up period. The median PFS of study population was 15.7 months (range, 2.3-33.8 months). Univariate analysis showed that male sex, heavy smoking (smoking intensity $\geq 30$ pack-years), poor ECOG PS, involvement of three or more organs, and presence of liver metastasis were associated with poor PFS (all $p<0.05$ ). In addition, high Romol expression was significantly associated with shorter PFS $(p=0.0165)$. Multivariate analysis showed that male sex (hazard ratio $[\mathrm{HR}]=2.09,95 \%$ confidence interval $[\mathrm{CI}]: 1.08-4.03)$, heavy smoking $(\mathrm{HR}=2.13$, 95\% CI: 1.02-4.45), poor ECOG PS ( $\mathrm{HR}=2.97,95 \% \mathrm{CI}: 1.53-5.75)$, presence of liver metastasis ( $\mathrm{HR}=4.25,95 \% \mathrm{CI}: 1.85-9.76)$, and high Romol ( $\mathrm{HR}=2.48,95 \% \mathrm{CI}: 1.35-4.56)$ were independently associated with shorter PFS. Patients with high Romol expression were likely to have poor PFS compared to those with low Romol expression as shown in Kaplan-Meier survival curves (Figure 2A).

\section{OS According to Romo1 Expression}

Table 4 shows the results of the OS analysis. The median OS of study population was 36.5 months (range, 3.5-52.8 months). Univariate analysis showed that heavy smoking, metastases involving three or more organs, and presence of liver metastasis were significantly associated with shorter OS (all $p<$ 0.05). In addition, high Romol expression was significantly associated with shorter OS $(p=0.0006)$. Multivariate analysis showed that heavy smoking $(\mathrm{HR}=2.77,95 \% \mathrm{CI}: 1.31-5.70)$, presence of liver metastasis ( $\mathrm{HR}=2.97,95 \% \mathrm{CI}: 1.24-7.14)$, and high Romol expression ( $\mathrm{HR}=3.17,95 \% \mathrm{CI}$ : 1.57-6.41) were independently associated with shorter OS. Patients with high Romol expression were likely to have poor OS compared to those with low Romol expression as shown in Kaplan-Meier survival curves (Figure 2B).

\section{Frequency of Acquired T790M Mutation According to Romo1 Expression}

To identify whether Romol expression is associated with the emergence of a secondary T790M mutation, we evaluated the mutation rates according to different Romo1 groups. Among 77 patients who progressed after first-line TKI therapy, 56 (72.7\%) underwent T790M mutation testing. The frequencies of T790M stratified by clinicopathological parameters of the 56 patients are 

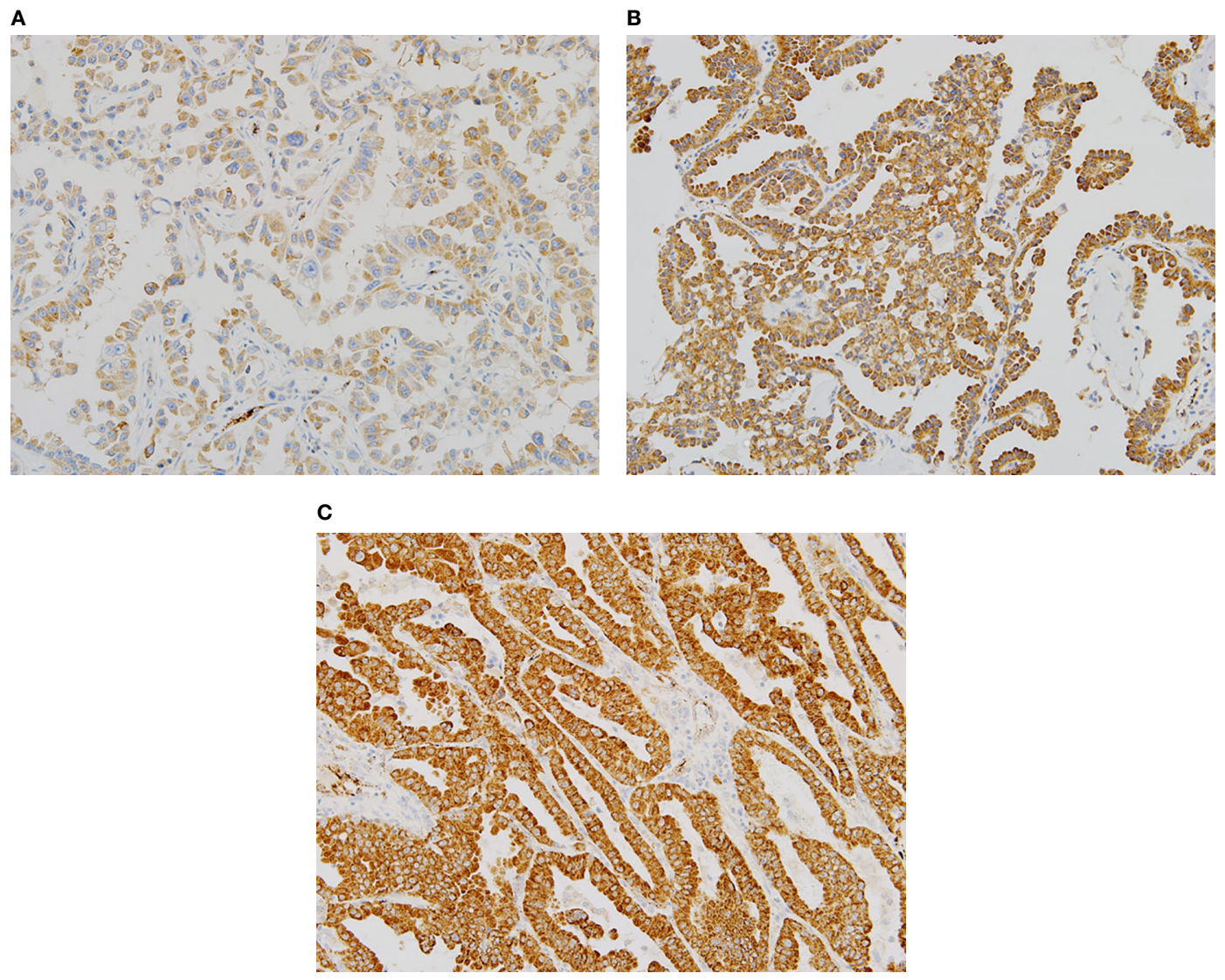

FIGURE 1 | Representative examples of immunochemical staining for reactive oxygen species modulator 1 with different histologic scores (H scores) ( $\times 200)$. Romo1 was primarily detected in the cytoplasm. (A) $\mathrm{H}$ score of 50; (B) $\mathrm{H}$ score of 150; and (C) $\mathrm{H}$ score of 250.

TABLE 2 | Treatment response according to different Romo1 expression.

No. of patients (\%)

p-value

\section{Low Romo1 $(\mathrm{n}=71)$}

High Romo1 $(n=25)$

\begin{tabular}{lcc}
\hline Response rate (CR+PR) & $60(84.5)$ & $17(68.0)$ \\
CR & $1(1.4)$ & $0(0)$ \\
PR & $59(83.1)$ & $17(68.0)$ \\
SD & $9(12.7)$ & $3(12.0)$ \\
PD & $2(2.8)$ & $5(20.0)$ \\
\hline
\end{tabular}

$C R$, complete response; $P R$, partial response; $S D$, stable disease; $P D$, progressive disease.

summarized in Table 5. Overall, 20 (20/56, 35.7\%) patients harbored the $\mathrm{T} 790 \mathrm{M}$ mutation. The mutation rates were significantly lower in patients with the L858R mutation and those who received TKI for less than 12 months (all $p<0.05$ ). In addition, patients with high Romol expression showed a significantly lower frequency of T790M mutation than those with low Romol expression ( $16.7 \%$ vs. $38.3 \%, p=0.0365$; Figure 3). Univariate analysis for the factors associated with the frequency of T790M mutation showed that L858R mutation, TKI use for less than 12 months, and high Romol expression were significantly associated with a lower incidence of T790M mutations (all $p<0.05$ ). Multivariate analysis showed that the
L858R mutation (odds ratio $[\mathrm{OR}]=0.46,95 \%$ CI: $0.08-0.94$ ), TKI use for less than 12 months (OR $=0.23$, 95\% CI: 0.05-0.89), and high Romol expression ( $\mathrm{OR}=0.36,95 \% \mathrm{CI}$ : 0.07-0.96) were independently associated with a low incidence of acquired T790M.

\section{DISCUSSION}

Our study demonstrated that high Romol expression was significantly associated with poor PFS and OS in patients with EGFR-mutant lung adenocarcinoma treated with frontline 
TABLE 3 | Progression-free survival according to clinicopathological parameters of all study subjects ( $n=96)$.

\begin{tabular}{|c|c|c|c|c|c|}
\hline & \multirow[t]{2}{*}{ Median PFS (months) } & \multicolumn{2}{|c|}{ Univariate } & \multicolumn{2}{|c|}{ Multivariate } \\
\hline & & HR (95\% Cl) & $p$-value & HR (95\% Cl) & $p$-value \\
\hline All & 15.7 & & & & \\
\hline Age & & & 0.2190 & NA & \\
\hline$<70$ & 15.7 & reference & & & \\
\hline$\geq 70$ & 15.0 & $1.36(0.83-2.22)$ & & & \\
\hline Sex & & & 0.0050 & & 0.0288 \\
\hline Male & 12.6 & $2.00(1.23-3.24)$ & & $2.09(1.08-4.03)$ & \\
\hline Female & 22.1 & reference & & reference & \\
\hline Smoking history & & & 0.0933 & & 0.4170 \\
\hline Never & 19.9 & reference & & $1.39(0.68-3.14)$ & \\
\hline Ever & 13.0 & $1.54(0.93-2.54)$ & & reference & \\
\hline Smoking intensity & & & 0.0210 & & 0.0450 \\
\hline <30 pack-years & 19.9 & reference & & reference & \\
\hline z30 pack-years & 11.2 & $1.95(1.11-3.45)$ & & $2.13(1.02-4.45)$ & \\
\hline ECOG PS & & & 0.0204 & & 0.0013 \\
\hline 0,1 & 20.1 & reference & & reference & \\
\hline$\geq 2$ & 12.1 & $1.92(1.11-3.32)$ & & $2.97(1.53-5.75)$ & \\
\hline Stage & & & 0.1050 & & 0.5860 \\
\hline III & 29.8 & $0.55(0.27-1.13)$ & & $1.27(0.54-3.01)$ & \\
\hline IV & 15.5 & reference & & reference & \\
\hline Involved organ & & & 0.0188 & & \\
\hline$<3$ & 19.9 & reference & & reference & \\
\hline$\geq 3$ & 12.4 & $1.90(1.11-3.24)$ & & $1.29(0.65-2.56)$ & 0.4622 \\
\hline Brain metastasis & & & 0.8982 & NA & \\
\hline No & 16.4 & reference & & & \\
\hline Yes & 15.7 & $1.03(0.62-1.71)$ & & & \\
\hline Liver metastasis & & & 0.0061 & & 0.0006 \\
\hline No & 19.9 & reference & & reference & \\
\hline Yes & 12.1 & $2.56(1.31-5.00)$ & & 4.25 (1.85-9.76) & \\
\hline EGFR subtypes* & & & 0.2850 & NA & \\
\hline 19del & 15.7 & 1.39 (0.15-2.85) & & & \\
\hline L858R & 15.3 & reference & & & \\
\hline First-line TKI & & & 0.0821 & & 0.1208 \\
\hline Gefitinib/erlotinib & 13.3 & $1.98(1.03-4.16)$ & & $1.45(0.88-7.10)$ & \\
\hline Afatinib & 18.5 & reference & & reference & \\
\hline Romo1 expression & & & 0.0165 & & 0.0034 \\
\hline Low $(<200)$ & 19.9 & reference & & reference & \\
\hline High ( $\geq 200)$ & 13.1 & 1.90 (1.13-3.22) & & $2.48(1.35-4.56)$ & \\
\hline
\end{tabular}

${ }^{*}$ Analysis of 91 patients, excluding 5 patients with uncommon or compound mutations.

ECOG PS, Eastern Cooperative Oncology Group Performance Status; EGFR, epidermal growth factor receptor; 19del, deletion mutation at exon 19; TKI, tyrosine kinase inhibitor; HR, hazard ratio; Cl, confidence interval; NA, not analyzed.

targeted therapy. In addition, it was associated with lower rate of secondary T790M mutations after TKI use. To the best of our knowledge, this is the first study to suggest the predictive and prognostic value of Romol in oncogene-driven malignancies. The current data highlight that high Romol expression might confer an aggressive phenotype, requiring tailored therapeutic approaches among EGFR-mutant tumors.

EGFR mutations are major driving genetic alterations that account for $15-50 \%$ of patients diagnosed with lung adenocarcinoma $(14,15)$. EGFR-TKIs are recommended as the first-line treatment in patients harboring these mutations as they improve both PFS and OS compared with those only treated with chemotherapy (16). However, the efficacy of EGFR-TKIs is still suboptimal, mainly because of the primary and secondary resistance, which is one of the major challenges in the management of these patients (17-19). Identification of the mechanisms leading to TKI resistance or prediction of treatment response before TKI use is critical to improve the clinical outcomes in this patient population. Although the resistant mechanism for EGFR-TKIs has been widely studied, it is not fully understood; T790M mutation, concurrent genetic alterations, MET amplification, epithelial-mesenchymal transition (EMT), and histological transformation are considered to be contributing factors $(16,17)$.

Cancer cells are characterized by increased ROS levels compared with normal cells, and the upregulated ROS are involved in various biological processes of carcinogenesis, including cell signaling, proliferation, and metastasis, as well as chemoresistance (18-20). Cumulating evidence indicates that ROS play a critical role in EGFR-TKI resistance. Increased oxidative stress induces TKI resistance of EGFR-mutant cells by activating phosphorylation and impairing the dimerization of EGFR $(21,22)$. In addition, ROS play a critical role in EMT engagement $(23,24)$ and interferon gamma-induced Src activation via the Raf/ERK signaling pathway (25). Moreover, chronic treatment with EGFR-TKIs enhances intracellular 
A

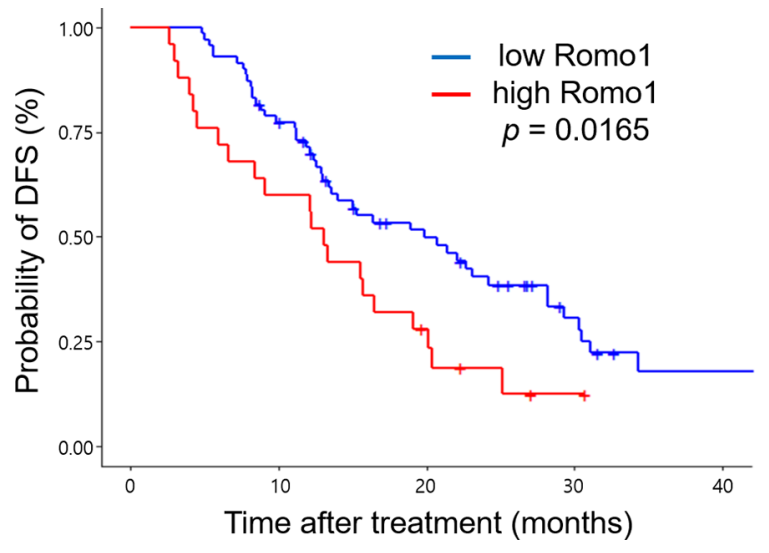

B

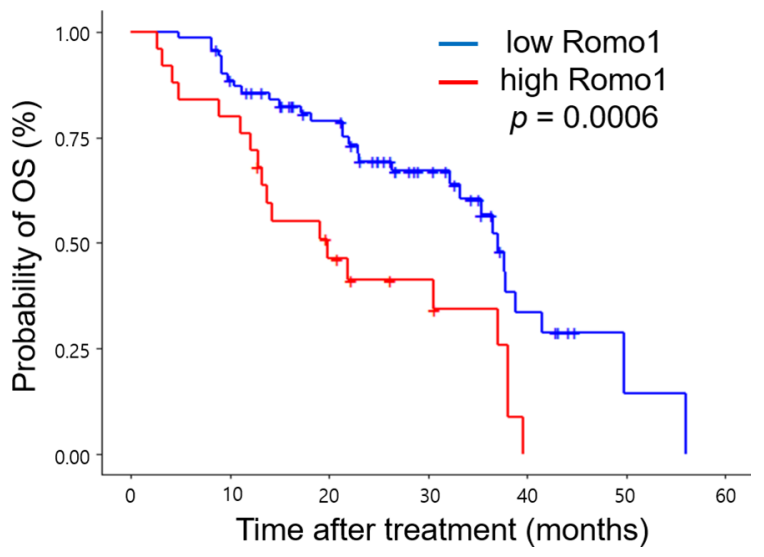

FIGURE 2 | Kaplan-Meier curves of progression-free survival (PFS, A) and overall survival (OS, B) according to different expression levels of Romo1. P-values were determined using the log-rank test.

oxidative stress through NOX4, and morphologic changes and dysfunction of mitochondria $(26,27)$. Antioxidants can alleviate TKI-resistance by inhibiting ROS-induced aberrant phosphorylation of EGFR and AKT $(26,28)$. Taken together, ROS are involved in both primary and secondary resistance to EGFR-TKIs via various mechanisms, including abnormal EGFR phosphorylation, EMT, and Sarc activation.

Romo1 is a major regulator of intracellular ROS production, and Romol-induced ROS are indispensable for the proliferation of normal and cancer cells $(6,7)$. Studies have consistently demonstrated the potential value of Romol as a novel biomarker for various lung cancer treatment $(11,12,29,30)$. Table 6 summarizes these previous studies and the present data, evaluating the clinical implications of Romol in lung cancer patients. Different studies have demonstrated different cutoffs from each other; however, the low $\mathrm{H}$ score cutoffs in two studies $(11,30)$ can be attributed to the early stage population enrolled in those studies. The present data suggest that Romol levels seem to be abundant in advanced disease. Based on the critical association between Romol and ROS production and the contribution of ROS in EGFR-TKI resistance, we hypothesized that Romol may be a novel biomarker in patients treated with targeted therapy. In addition to the predictive and prognostic values of Romo1, our present data suggest the possibility of this novel protein as a potential treatment target for EGFR-mutant NSCLC, considering the crucial association between ROS and TKI resistance. To address this issue, we have just started a preclinical study on the regulation mechanism of Romol, combinational effect of Romol inhibition and EGFR-TKIs, and drug screening for its inhibitors.

Recent studies have demonstrated the clinical usefulness of Romol gene expression and the novel association between micro RNA and Romol. Alizamir et al. demonstrated that Romol mRNA expression increased in bladder cancer tissues and that Romol overexpression was associated with advanced tumor (31). Similarly, Afshar et al. showed that Romol mRNA was overexpressed in gastric cancer tissues and that its overexpression was associated with unfavorable prognosis in patients with gastric cancer (32). Moreover, Wang et al. reported that LINC00319, a long non coding RNA, is correlated with early recurrence of bladder cancer, and that LINC00319 is a sponge for miR-4492, which directly targets Romol expression. In that study, the miR-4492/Romol axis was shown to regulate proliferation, migration, and tumor invasion of bladder cancer cells (33). These findings suggest that evaluating Romol gene expression could be more useful than evaluating protein expression using IHC because the former can be less variable than the latter. In addition, the potential role of micro RNA as a regulator of Romol in various human malignancies should be investigated in future studies.

Secondary T790M mutation is a major resistance mechanism and has been identified in approximately half of patients who progressed after EGFR-TKI therapy (34). Detection of this mutation is important for the management of EGFR-mutant patients because T790M-positive tumors are susceptible to thirdgeneration EGFR-TKIs, such as osimertinib. Although mutational analysis using tumor tissue or body fluid is recommended, it is often limited by factors, such as the invasiveness of tissue acquisition and the insufficiency or inaccessibility of tissue samples. Previous studies have demonstrated that the frequent T790M mutation is associated with 19del and long-term exposure to EGFR-TKIs (35-37). Our present data confirmed these previous findings, and additionally suggest that high Romol expression could be a negative predictor of T790M development after frontline TKI. The reason that Romol is associated with the emergence of the T790M mutation is unclear; however, it may be attributed to the different durations of treatment with EGFR-TKI according to different Romol expression. As suggested in the previous studies, T790M-positive cells undergo selection and are enriched during EGFR-TKI treatment (38-40). In addition, time is required for the development of T790M-dominant tumor because cells 
TABLE 4 | Overall survival according to clinicopathological parameters of all study subjects ( $n=96)$.

\begin{tabular}{|c|c|c|c|c|c|}
\hline & \multirow[t]{2}{*}{ Median OS (months) } & \multicolumn{2}{|c|}{ Univariate } & \multicolumn{2}{|c|}{ Multivariate } \\
\hline & & HR (95\% Cl) & $p$-value & HR (95\% Cl) & ${ }^{*} p$-value \\
\hline All & 36.5 & & & & \\
\hline Age & & & & NA & \\
\hline$<70$ & 36.5 & reference & & & \\
\hline$\geq 70$ & 35.4 & $1.42(0.8-2.51)$ & 0.2321 & & \\
\hline Sex & & & & & 0.5032 \\
\hline Male & 32.2 & $1.60(0.89-2.87)$ & 0.1147 & $1.31(0.59-2.89)$ & \\
\hline Female & 37.1 & reference & & reference & \\
\hline Smoking History & & & 0.0750 & & 0.0619 \\
\hline Never & 37.1 & reference & & reference & \\
\hline Ever & 32.2 & $1.71(0.95-3.10)$ & & $2.22(0.96-5.1)$ & \\
\hline Smoking intensity & & & 0.0068 & & 0.0157 \\
\hline <30 pack-years & 37.0 & reference & & reference & \\
\hline$\geq 30$ pack-years & 32.2 & $2.31(1.26-4.24)$ & & $2.77(1.31-5.70)$ & \\
\hline ECOG PS & & & 0.4443 & NA & \\
\hline 0,1 & 36.5 & reference & & & \\
\hline$\geq 2$ & 33.2 & $1.28(0.68-2.4)$ & & & \\
\hline Stage & & & 0.8138 & NA & \\
\hline III & 30.5 & reference & & & \\
\hline IV & 33.2 & 1.09 (0.52-2.28) & & & \\
\hline Involved organ & & & 0.0048 & & 0.0862 \\
\hline$<3$ & 37.1 & reference & & reference & \\
\hline$\geq 3$ & 21.4 & $2.41(1.31-4.44)$ & & $2.00(0.91-4.41)$ & \\
\hline Brain metastasis & & & 0.9999 & NA & \\
\hline No & 36.5 & reference & & & \\
\hline Yes & 35.4 & $1.04(0.56-1.79)$ & & & \\
\hline Liver metastasis & & & 0.0008 & & 0.0148 \\
\hline No & 36.5 & reference & & reference & \\
\hline Yes & 22.0 & $2.93(1.57-5.51)$ & & $2.97(1.24-7.16)$ & \\
\hline EGFR subtypes* & & & 0.7684 & NA & \\
\hline 19del & 37.0 & $1.23(0.32-4.73)$ & & & \\
\hline L858R & 33.2 & reference & & & \\
\hline First-line TKIs & & & & & 0.2019 \\
\hline Gefitinib/erlotinib & 30.2 & $1.70(0.95-3.57)$ & 0.0847 & $1.41(0.69-3.27)$ & \\
\hline Afatinib & 36.1 & reference & & reference & \\
\hline Romo1 expression & & & 0.0006 & & 0.0013 \\
\hline Low $(<200)$ & 37.0 & reference & & reference & \\
\hline High ( $\geq 200)$ & 19.8 & $2.77(1.55-4.96)$ & & $3.17(1.57-6.41)$ & \\
\hline
\end{tabular}

*Analysis for 91 patients excluding 5 patients with uncommon or compound mutations.

ECOG PS, Eastern Cooperative Oncology Group Performance Status; EGFR, epidermal growth factor receptor; 19del, deletion mutation at exon 19; TKI, tyrosine kinase inhibitor; HR, hazard ratio; Cl, confidence interval; NA, not analysed.

harboring the mutation grow slowly compared with T790Mnegative counterparts (41). Based on these findings, we hypothesize that shorter treatment duration may be the cause of lower frequency of the T790M mutation in high Romo1 population. Our hypothesis should be elucidated through further investigations.

The present data suggest that Romol overexpression confers a distinct aggressive phenotype that may require different treatment strategies. Because TKI monotherapy could be less effective in patients with high Romol expression, other therapeutic options, such as combination with chemotherapy or immunotherapy, can be considered. A recent study showed that pemetrexed-based doublet combined with gefitinib showed better RR ( $84 \%$ vs. $67 \%, p<0.001$ ), longer PFS (20.9 vs. 11.9 months, $\mathrm{HR}=0.49 ; p<0.001$ ), and OS (50.9 vs. 38.8 months, HR $=0.722 ; p=0.021)$ compared with gefitinib monotherapy in EGFR-mutant tumors (42). To prove our hypothesis and determine the optimal treatment strategy for those with different Romo1 expression levels, large-scale prospective investigations are required.

This study had several limitations. First, it is a relatively small retrospective study performed at a single institute, and the slides were reviewed by a single pathologist. Second, we did not include nutritional status or comorbidities as clinical characteristics, which might influence the survival outcome of our patient population. To overcome these potential caveats, we aimed to enhance the validity of our data by long-term follow-up. Third, tumors harboring other driver genetic alterations, such as ROS1 and $A L K$ fusions, were excluded because of their low frequency in our patient cohort. Fourth, the interval changes in Romo1 expression in patients with progressive disease after TKI failure were not investigated. As mentioned earlier, EGFR-TKIs can induce ROS production, and thus, Romol expression can be affected by previous treatments. The predicted value or impact of 
TABLE 5 | Analysis for the factors associated with emergence of secondary T790M mutation ( $n=56)$.

\begin{tabular}{|c|c|c|c|c|c|c|c|}
\hline & \multicolumn{2}{|c|}{ T790M } & \multirow[t]{2}{*}{$p$-value* } & \multicolumn{2}{|c|}{ Univariate analysis } & \multicolumn{2}{|c|}{ Multivariate analysis } \\
\hline & Negative $(n=36)$ & Positive $(n=20)$ & & OR (95\% Cl) & p-value & OR $(95 \% \mathrm{Cl})$ & $p$-value \\
\hline \multicolumn{8}{|l|}{ All } \\
\hline Age & & & 0.0987 & & 0.0926 & & 0.1368 \\
\hline$<70$ & 15 (55.6) & $12(44.4)$ & & $0.54(0.12-1.31)$ & & $0.35(0.17-1.92)$ & \\
\hline$\geq 70$ & $21(75.0)$ & $8(25.0)$ & & reference & & reference & \\
\hline Sex & & & 0.6895 & & 0.6897 & NA & \\
\hline Male & $19(65.5)$ & $10(34.5)$ & & reference & & & \\
\hline Female & $17(63.0)$ & $10(27.0)$ & & $0.81(0.28-2.30)$ & & & \\
\hline Smoking History & & & 0.0781 & & 0.0857 & & 0.0565 \\
\hline Never & $19(54.3)$ & $16(45.7)$ & & $0.34(0.10-1.17)$ & & $0.23(0.05-1.04)$ & \\
\hline Ever & $17(80.9)$ & $4(19.1)$ & & reference & & reference & \\
\hline Smoking intensity & & & 0.3558 & & 0.2735 & NA & \\
\hline$<30$ pack-years & $26(60.5)$ & 17 (39.5) & & $0.46(0.12-1.84)$ & & & \\
\hline$\geq 30$ pack-years & $10(79.6)$ & $3(20.4)$ & & reference & & & \\
\hline ECOG PS & & & 0.1136 & & 0.0989 & & 0.1105 \\
\hline 0,1 & $28(70.0)$ & $12(30.0)$ & & reference & & reference & \\
\hline$\geq 2$ & $8(50.0)$ & $8(50.0)$ & & $0.41(0.13-1.26)$ & & $0.34(0.09-1.28)$ & \\
\hline Stage & & & 0.2458 & & 0.8063 & NA & \\
\hline III & $4(57.1)$ & $3(42.9)$ & & $0.83(0.19-3.71)$ & & & \\
\hline IV & $31(64.6)$ & $17(35.4)$ & & reference & & & \\
\hline Involved organ & & & 0.9861 & & 0.9861 & NA & \\
\hline$<3$ & $25(64.1)$ & $14(35.9)$ & & reference & & & \\
\hline$\geq 3$ & $11(64.7)$ & 6 (35.3) & & $0.99(0.32-3.10)$ & & & \\
\hline Brain metastasis & & & 0.2301 & & 0.2333 & NA & \\
\hline No & $25(69.4)$ & $11(30.6)$ & & reference & & & \\
\hline Yes & $11(22.0)$ & $9(78.0)$ & & $0.52(0.18-1.53)$ & & & \\
\hline Liver metastasis & & & 0.7210 & & 0.6068 & NA & \\
\hline No & $30(65.2)$ & $16(34.8)$ & & reference & & & \\
\hline Yes & $6(60.0)$ & $4(40.0)$ & & $0.70(0.18-2.72)$ & & & \\
\hline EGFR subtypes & & & 0.0460 & & 0.0339 & & 0.0413 \\
\hline 19del & $11(52.3)$ & $10(47.7)$ & & reference & & reference & \\
\hline L858R & $25(75.7)$ & $8(24.3)$ & & $0.29(0.09-0.91)$ & & $0.46(0.08-0.94)$ & \\
\hline First-line $\mathrm{TK}^{\star}$ & & & 0.0846 & & 0.0629 & & 0.2614 \\
\hline Gefitinib/erlotinib & $11(55.0)$ & $8(45.0)$ & & reference & & reference & \\
\hline Afatinib & $24(66.7)$ & $12(33.3)$ & & $0.50(0.21-1.78)$ & & $0.65(0.12-1.39)$ & \\
\hline Duration of TKI use & & & 0.0318 & & 0.0457 & & 0.0411 \\
\hline$<12$ months & $17(80.9)$ & $4(19.1)$ & & $0.24(0.10-0.91)$ & & $0.23(0.05-0.89)$ & \\
\hline$\geq 12$ months & $20(57.1)$ & $15(42.9)$ & & reference & & reference & \\
\hline Romo1 expression & & & 0.0365 & & 0.0401 & & 0.0459 \\
\hline Low $(<200)$ & $22(61.1)$ & 17 (38.3) & & $0.28(0.07-0.98)$ & & $0.36(0.07-0.96)$ & \\
\hline High ( $\geq 200)$ & 14 (82.3.) & $3(16.7)$ & & reference & & reference & \\
\hline
\end{tabular}

*Analysis after excluding 2 patients with uncommon or compound mutations.

ECOG PS, Eastern Cooperative Oncology Group Performance Status; EGFR, epidermal growth factor receptor; 19del, deletion mutation at exon 19; TKI, tyrosine kinase inhibitor; OR, odds ratio; $\mathrm{Cl}$, confidence interval; NA, not analysed.

TABLE 6 | Summary of studies on the predictive and prognostic value of Romo1 in lung cancer.

\begin{tabular}{|c|c|c|c|c|c|c|}
\hline Author, year & Patients number & Treatment setting & Sample used & Quantification method & Cut-off H score & $\begin{array}{l}\text { Association with Romo1 } \\
\text { expression }\end{array}$ \\
\hline Lee et al., 2015 (11) & 110 & Surgery & Tissue & IHC with H score & 159 & DFS, OS \\
\hline Lee et al., 2017 (12) & 88 & Chemotherapy & Tissue & IHC with H score & 200 & PFS, OS \\
\hline $\begin{array}{l}\text { Kong et al., } 2019 \\
\text { (29) }\end{array}$ & 49 & Definitive radiotherapy & Tissue & $\mathrm{IHC}$ with $\mathrm{H}$ score & 200 & PFS, OS \\
\hline $\begin{array}{l}\text { Kong et al., } 2020 \\
\text { (30) }\end{array}$ & 40 & $\begin{array}{l}\text { Stereotactic } \\
\text { radiosurgery }\end{array}$ & Tissue & IHC with $\mathrm{H}$ score & 125 & DMFS \\
\hline The present study & 96 & EGFR-TKI & Tissue & $\mathrm{IHC}$ with $\mathrm{H}$ score & 200 & $\begin{array}{c}\text { PFS, OS, frequency of T790M } \\
\text { mutation }\end{array}$ \\
\hline
\end{tabular}

IHC, immunohistochemistry; H score, Histologic score; DFS, disease-free survival; PFS, progression-free survival; OS, overall survival; EGFR, epidermal growth factor receptor; TKI, tyrosine kinase inhibitor; DMFS, Distant metastasis-free survival. 


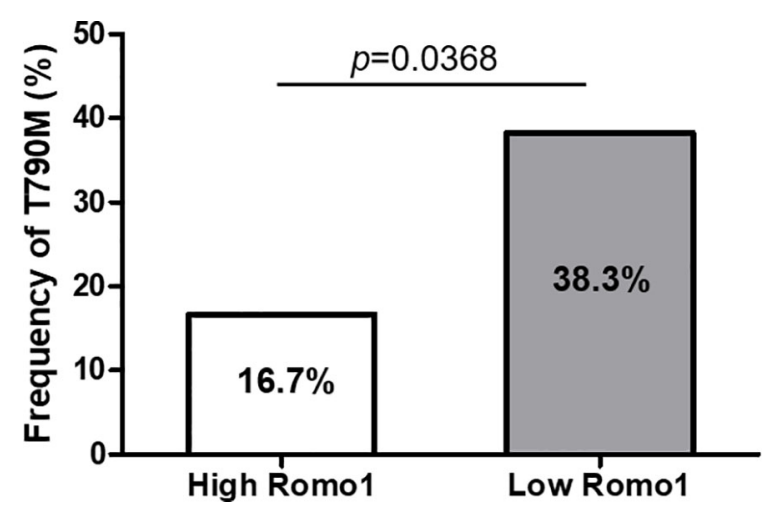

FIGURE 3 | Frequency of secondary T790M mutation after tyrosine kinase inhibitor (TKI) failure according to different expression levels of Romo1. The rate of T790M mutation is significantly lower in the high Romo1 group compared with the low Romo1 group ( $p=0.0369$ ).

the dynamics of Romol expression on subsequent treatment could be an interesting topic for future studies. Finally, we did not simultaneously explore other factors that could be associated with TKI resistance, including immune phenotypes or coexisting genetic alterations. We are currently working on a possible association between Romol expression and immunologic signatures or concurrent genetic alterations in EGFR-mutant populations.

\section{CONCLUSION}

The present data demonstrate that high Romol expression is associated with unfavorable clinical outcomes, not only due to the poor response to first-line TKI treatment but also because of the less emergence of secondary T790M mutation. These findings highlight that Romol overexpression might confer a distinct aggressive phenotype among EGFR-mutant tumors, requiring a different therapeutic approach, although further studies are needed to verify our results. In addition, future studies should focus on whether Romol has similar clinical implications in other malignancies harboring driver genetic alterations, and optimal treatment strategy for the Romo1overexpressed, EGFR-mutant tumors. Large prospective studies validating our data might facilitate the clinical use of Romo1 expression for the risk stratification and prediction of prognosis in those patient population.

\section{REFERENCES}

1. Sung H, Ferlay J, Siegel RL, Laversanne M, Soerjomataram I, Jemal A, et al. Global Cancer Statistics 2020: GLOBOCAN Estimates of Incidence and Mortality Worldwide for 36 Cancers in 185 Countries. CA Cancer J Clin (2021) 71(3):209-49. doi: 10.3322/caac.21660

2. Kim HC, Jung CY, Cho DG, Jeon JH, Lee JE, Ahn JS, et al. Clinical Characteristics and Prognostic Factors of Lung Cancer in Korea: A Pilot Study of Data From the Korean Nationwide Lung Cancer Registry. Tuberc Respir Dis (Seoul) (2019) 82(2):118-25. doi: 10.4046/trd.2017.0128

\section{DATA AVAILABILITY STATEMENT}

The original contributions presented in the study are included in the article/Supplementary Material. Further inquiries can be directed to the corresponding author.

\section{ETHICS STATEMENT}

The studies involving human participants were reviewed and approved by Kyung Hee University Hospital. The patients/ participants provided their written informed consent to participate in this study.

\section{AUTHOR CONTRIBUTIONS}

SHL conceived and designed the study. WGK, J-YS, and SHL collected, analyzed, and interpreted the data, and drafted the article. SHL revised the paper critically. All authors contributed to the article and approved the submitted version.

\section{FUNDING}

This research was supported by a grant from the Basic Research Program through the National Research Foundation funded by the Ministry of Science and ICT (2019R1F1A1041812), and a grant of the Korea Health Technology R\&D Project through the Korea Health Industry Development Institute (KHIDI) funded by the Ministry of Health and Welfare (HI18C1944) of the Republic of Korea.

\section{ACKNOWLEDGMENTS}

We would like to thank Editage (www.editage.co.kr) for English language editing.

\section{SUPPLEMENTARY MATERIAL}

The Supplementary Material for this article can be found online at: https://www.frontiersin.org/articles/10.3389/fonc.2021. 770230/full\#supplementary-material

3. Kim HC, Choi CM. Current Status of Immunotherapy for Lung Cancer and Future Perspectives. Tuberc Respir Dis (Seoul) (2020) 83(1):14-9. doi: $10.4046 / \operatorname{trd} .2019 .0039$

4. Garon EB, Hellmann MD, Rizvi NA, Carcereny E, Leighl NB, Ahn MJ, et al. Five-Year Overall Survival for Patients With Advanced Non-Small-Cell Lung Cancer Treated With Pembrolizumab: Results From the Phase I KEYNOTE-001 Study. J Clin Oncol (2019) 37(28):2518-27. doi: 10.1200/ JCO.19.00934

5. Yoo YA, Kim MJ, Park JK, Chung YM, Lee JH, Chi SG, et al. Mitochondrial Ribosomal Protein L41 Suppresses Cell Growth in Association With P53 and 
p27Kip1. Mol Cell Biol (2005) 25(15):6603-16. doi: 10.1128/MCB.25.15.66036616.2005

6. Shin JA, Chung JS, Cho SH, Kim HJ, Yoo YD. Romol Expression Contributes to Oxidative Stress-Induced Death of Lung Epithelial Cells. Biochem Biophys Res Commun (2013) 439(2):315-20. doi: 10.1016/j.bbrc.2013.07.012

7. Na AR, Chung YM, Lee SB, Park SH, Lee MS, Yoo YD. A Critical Role for Romo1-Derived ROS in Cell Proliferation. Biochem Biophys Res Commun (2008) 369(2):672-8. doi: 10.1016/j.bbrc.2008.02.098

8. Hwang IT, Chung YM, Kim JJ, Chung JS, Kim BS, Kim HJ, et al. Drug Resistance to 5-FU Linked to Reactive Oxygen Species Modulator 1. Biochem Biophys Res Commun (2007) 359(2):304-10. doi: 10.1016/j.bbrc.2007.05.088

9. Chung JS, Park S, Park SH, Park ER, Cha PH, Kim BY, et al. Overexpression of Romol Promotes Production of Reactive Oxygen Species and Invasiveness of Hepatic Tumor Cells. Gastroenterology (2012) 143(4):1084-94.e7. doi: 10.1053 /j.gastro.2012.06.038

10. Lee S, Park YH, Chung JS, Yoo YD. Romol and the NF-kappaB Pathway are Involved in Oxidative Stress-Induced Tumor Cell Invasion. Int J Oncol (2015) 46(5):2021-8. doi: 10.3892/ijo.2015.2889

11. Lee SH, Min JW, Lee JS, Kim CH, Yoo YD, Lee EJ, et al. Reactive Oxygen Species Modulator 1 (Romo1) Overexpression is an Independent Predictor of Poor Survival in NSCLC Patients Who Undergo Surgical Resection. Lung Cancer (2015) 87(1):45-52. doi: 10.1016/j.lungcan.2014.11.004

12. Lee SH, Choi SI, Lee JS, Kim CH, Jung WJ, Lee EJ, et al. Reactive Oxygen Species Modulator 1 (Romol) Predicts Poor Outcomes in Advanced NonSmall Cell Lung Cancer Patients Treated With Platinum-Based Chemotherapy. Cancer Res Treat (2017) 49(1):141-9. doi: 10.4143/ crt.2016.133

13. Kim HJ, Jo MJ, Kim BR, Kim JL, Jeong YA, Na YJ, et al. Reactive Oxygen Species Modulator-1 (Romo1) Predicts Unfavorable Prognosis in Colorectal Cancer Patients. PloS One (2017) 12(5):e0176834. doi: 10.1371/ journal.pone.0176834

14. Goldstraw P, Chansky K, Crowley J, Rami-Porta R, Asamura H, Eberhardt WE, et al. The IASLC Lung Cancer Staging Project: Proposals for Revision of the TNM Stage Groupings in the Forthcoming (Eighth) Edition of the TNM Classification for Lung Cancer. J Thorac Oncol (2016) 11(1):39-51. doi: 10.1016/j.jtho.2015.09.009

15. Eisenhauer EA, Therasse P, Bogaerts J, Schwartz LH, Sargent D, Ford R, et al. New Response Evaluation Criteria in Solid Tumours: Revised RECIST Guideline (Version 1.1). Eur J Cancer (2009) 45(2):228-47. doi: 10.1016/ j.ejca.2008.10.026

16. Kim IA, Lee JS, Kim HJ, Kim WS, Lee KY. Cumulative Smoking Dose Affects the Clinical Outcomes of EGFR-Mutated Lung Adenocarcinoma Patients Treated With EGFR-TKIs: A Retrospective Study. BMC Cancer (2018) 18 (1):768. doi: 10.1186/s12885-018-4691-0

17. Nagano T, Tachihara M, Nishimura Y. Mechanism of Resistance to Epidermal Growth Factor Receptor-Tyrosine Kinase Inhibitors and a Potential Treatment Strategy. Cells (2018) 7(11):212. doi: 10.3390/cells7110212

18. Laurent A, Nicco C, Chereau C, Goulvestre C, Alexandre J, Alves A, et al. Controlling Tumor Growth by Modulating Endogenous Production of Reactive Oxygen Species. Cancer Res (2005) 65(3):948-56.

19. Brozovic A, Ambriovic-Ristov A, Osmak M. The Relationship Between Cisplatin-Induced Reactive Oxygen Species, Glutathione, and BCL-2 and Resistance to Cisplatin. Crit Rev Toxicol (2010) 40(4):347-59. doi: 10.3109/ 10408441003601836

20. Pluchino LA, Choudhary S, Wang HC. Reactive Oxygen Species-Mediated Synergistic and Preferential Induction of Cell Death and Reduction of Clonogenic Resistance in Breast Cancer Cells by Combined Cisplatin and FK228. Cancer Lett (2016) 381(1):124-32. doi: 10.1016/j.canlet. 2016.07.036

21. Filosto S, Khan EM, Tognon E, Becker C, Ashfaq M, Ravid T, et al. EGF Receptor Exposed to Oxidative Stress Acquires Abnormal Phosphorylation and Aberrant Activated Conformation That Impairs Canonical Dimerization. PloS One (2011) 6(8):e23240. doi: 10.1371/journal.pone. 0023240

22. Filosto S, Becker CR, Goldkorn T. Cigarette Smoke Induces Aberrant EGF Receptor Activation That Mediates Lung Cancer Development and Resistance to Tyrosine Kinase Inhibitors. Mol Cancer Ther (2012) 11(4):795-804. doi: 10.1158/1535-7163.MCT-11-0698
23. Giannoni E, Parri M, Chiarugi P. EMT and Oxidative Stress: A Bidirectional Interplay Affecting Tumor Malignancy. Antioxid Redox Signal (2012) 16 (11):1248-63. doi: 10.1089/ars.2011.4280

24. Nikitovic D, Corsini E, Kouretas D, Tsatsakis A, Tzanakakis G. ROS-Major Mediators of Extracellular Matrix Remodeling During Tumor Progression. Food Chem Toxicol (2013) 61:178-86. doi: 10.1016/j.fct.2013.06.013

25. Zibara K, Zeidan A, Bjeije H, Kassem N, Badran B, El-Zein N. ROS Mediates Interferon Gamma Induced Phosphorylation of Src, Through the Raf/ERK Pathway, in MCF-7 Human Breast Cancer Cell Line. J Cell Commun Signal (2017) 11(1):57-67. doi: 10.1007/s12079-016-0362-6

26. Okon IS, Coughlan KA, Zhang M, Wang Q, Zou MH. Gefitinib-Mediated Reactive Oxygen Specie (ROS) Instigates Mitochondrial Dysfunction and Drug Resistance in Lung Cancer Cells. J Biol Chem (2015) 290(14):9101-10. doi: 10.1074/jbc.M114.631580

27. Orcutt KP, Parsons AD, Sibenaller ZA, Scarbrough PM, Zhu Y, Sobhakumari A, et al. Erlotinib-Mediated Inhibition of EGFR Signaling Induces Metabolic Oxidative Stress Through NOX4. Cancer Res (2011) 71(11):3932-40. doi: 10.1158/0008-5472.CAN-10-3425

28. Zhang L, Li J, Hu J, Li D, Wang X, Zhang R, et al. Cigarette Smoke Extract Induces EGFR-TKI Resistance via Promoting EGFR Signaling Pathway and ROS Generation in NSCLC Cell Lines. Lung Cancer (2017) 109:109-16. doi: 10.1016/j.lungcan.2017.05.011

29. Kong M, Sung JY, Lee SH. Reactive Oxygen Species Modulator 1 As An Adverse Prognostic Marker In Stage III Non-Small Cell Lung Cancer Treated With Radiotherapy: A Retrospective Pilot Study. Onco Targets Ther (2019) 12:8263-73. doi: 10.2147/OTT.S217514

30. Kong M, Sung JY, Lee SH. Reactive Oxygen Species Modulator 1 is Associated With Poor Survival in Patients With Non-Small Cell Lung Cancer After Stereotactic Fractionated Radiosurgery: A Retrospective Pilot Study. Onco Targets Ther (2020) 13:8173-80. doi: 10.2147/OTT.S266344

31. Ghasemi H, Amini MA, Pegah A, Azizi E, Tayebinia H, Khanverdilou S, et al. Overexpression of Reactive Oxygen Species Modulator 1 Is Associated With Advanced Grades of Bladder Cancer. Mol Biol Rep (2020) 47(9):6497-505. doi: 10.1007/s11033-020-05702-1

32. Amini MA, Karimi J, Khodadadi I, Tavilani H, Talebi SS, Afshar B. Overexpression of ROMO1 and OMA1 Are Potentially Biomarkers and Predict Unfavorable Prognosis in Gastric Cancer. $J$ Gastrointest Cancer (2020) 51(3):939-46. doi: 10.1007/s12029-019-00330-w

33. Yang Y, Zhang F, Huang H, Xie Z, Huang W, Xie H, et al. Long Noncoding RNA LINC00319 Regulates ROMO1 Expression and Promotes Bladder Cancer Progression via miR-4492/ROMO1 Axis. J Cell Physiol (2020) 235 (4):3768-75. doi: 10.1002/jcp.29271

34. Chang YS, Choi CM, Lee JC. Mechanisms of Epidermal Growth Factor Receptor Tyrosine Kinase Inhibitor Resistance and Strategies to Overcome Resistance in Lung Adenocarcinoma. Tuberc Respir Dis (Seoul) (2016) 79 (4):248-56. doi: 10.4046/trd.2016.79.4.248

35. Nosaki K, Satouchi M, Kurata T, Yoshida T, Okamoto I, Katakami N, et al. Re-Biopsy Status Among Non-Small Cell Lung Cancer Patients in Japan: A Retrospective Study. Lung Cancer (2016) 101:1-8. doi: 10.1016/j.lungcan.2016.07.007

36. Matsuo N, Azuma K, Sakai K, Hattori S, Kawahara A, Ishii H, et al. Association of EGFR Exon 19 Deletion and EGFR-TKI Treatment Duration With Frequency of T790M Mutation in EGFR-Mutant Lung Cancer Patients. Sci Rep (2016) 6:36458. doi: 10.1038/srep36458

37. Goag EK, Lee JM, Chung KS, Kim SY, Leem AY, Song JH, et al. Usefulness of Bronchoscopic Rebiopsy of Non-Small Cell Lung Cancer With Acquired Resistance to Epidermal Growth Factor Receptor-Tyrosine Kinase Inhibitor. J Cancer (2018) 9(6):1113-20. doi: 10.7150/jca.21650

38. Chmielecki J, Foo J, Oxnard GR, Hutchinson K, Ohashi K, Somwar R, et al. Optimization of Dosing for EGFR-Mutant Non-Small Cell Lung Cancer With Evolutionary Cancer Modeling. Sci Transl Med (2011) 3(90):90ra59. doi: $10.1126 /$ scitranslmed.3002356

39. Iwama E, Takayama K, Harada T, Okamoto I, Ookubo F, Kishimoto J, et al. Highly Sensitive and Quantitative Evaluation of the EGFR T790M Mutation by Nanofluidic Digital PCR. Oncotarget (2015) 6(24):20466-73. doi: 10.18632/ oncotarget. 4058

40. Zheng D, YeX, Zhang MZ, Sun Y, Wang JY, Ni J, et al. Plasma EGFR T790M ctDNA Status is Associated With Clinical Outcome in Advanced NSCLC Patients With Acquired EGFR-TKI Resistance. Sci Rep (2016) 6:20913. doi: 10.1038/srep20913 
41. Hata A, Katakami N, Yoshioka H, Takeshita J, Tanaka K, Nanjo S, et al. Rebiopsy of Non-Small Cell Lung Cancer Patients With Acquired Resistance to Epidermal Growth Factor Receptor-Tyrosine Kinase Inhibitor: Comparison Between T790M Mutation-Positive and Mutation-Negative Populations. Cancer (2013) 119(24):4325-32. doi: 10.1002/cncr.28364

42. Hosomi Y, Morita S, Sugawara S, Kato T, Fukuhara T, Gemma A, et al. Gefitinib Alone Versus Gefitinib Plus Chemotherapy for Non-Small-Cell Lung Cancer With Mutated Epidermal Growth Factor Receptor: NEJ009 Study. J Clin Oncol (2020) 38(2):115-23. doi: 10.1200/JCO.19.01488

Conflict of Interest: The authors declare that the research was conducted in the absence of any commercial or financial relationships that could be construed as a potential conflict of interest.
Publisher's Note: All claims expressed in this article are solely those of the authors and do not necessarily represent those of their affiliated organizations, or those of the publisher, the editors and the reviewers. Any product that may be evaluated in this article, or claim that may be made by its manufacturer, is not guaranteed or endorsed by the publisher.

Copyright (C) 2021 Kwack, Sung and Lee. This is an open-access article distributed under the terms of the Creative Commons Attribution License (CC BY). The use, distribution or reproduction in other forums is permitted, provided the original author(s) and the copyright owner(s) are credited and that the original publication in this journal is cited, in accordance with accepted academic practice. No use, distribution or reproduction is permitted which does not comply with these terms. 